\title{
Rinosinusitis alérgica por Curvularia inaequalis (Shear) Boedijn
}

\author{
Rodrigo Cruz, Elizabeth Barthel y Jaime Espinoza
}

\section{Allergic rhinosinusitis by Curvularia inaequalis (Shear) Boedijn}

Curvularia inaequalis (Shear) Boedijn is a fungus dematiaceo, saprophyte and plant pathogen found mainly in tropical and subtropical areas, associated with various organic substrates. Rarely been identified in systemic infections, skin and there is only one report of allergic rhinosinusitis described above. A case of allergic fungal rhinosinusitis by Curvularia inaequalis (Shear) Boedijn in which diagnosis was considered the signs and symptoms, sinus CT and cultivation of mucin. The patient was treated with endoscopic surgical toilet, plus use of inhaled steroids and itraconazole systemic. With good clinical response, is asymptomatic at one year.

Key words: Allergic fungal rhinosinusitis, Curvularia inaequalis.

Palabras clave: Rinosinusitis alérgica fúngica, Curvularia inaequalis.

\section{Introducción}

$\mathrm{L}$ a rinosinusitis fúngica alérgica (RSFA) fue descrita inicialmente por Millar y Lamb en 1981, siendo denominada "aspergilosis sinusal alérgica" por su similitud histopatológica con la "aspergilosis broncopulmonar alérgica" $"$. Estudios posteriores demostraron que la mayoría de estos casos eran causados por especies distintas a Aspergillus, por lo que actualmente se le denomina "sinusitis fúngica alérgica" ${ }^{3,4}$. Los pacientes con esta infección, con frecuencia tienen compromiso unilateral y múltiple de los senos paranasales, presentando generalmente varias recurrencias en el tiempo. Dentro de los factores de riesgo conocidos se encuentra el antecedente de atopia, poliposis nasal, desviación septal u obstrucción del complejo osteomeatal ${ }^{4}$. Uno de los signos característicos de este cuadro es la secreción nasal mucosa espesa, de color amarillento verdoso, la cual recibe el nombre de mucina alérgica. En estudios histopatológicos se observa como un exudado rico en eosinófilos, linfocitos y cristales de Charcot-Leyden, los que corresponden al producto de la degradación de eosinófilos ${ }^{5,6}$.

Los agentes aislados con mayor frecuencia en esta patología son especies pertenecientes a los géneros Curvularia Boedijn, Bipolaris Shoemaker, Alternaria Nees ex Fries, Aspergillus Micheli ex Link ${ }^{6}$. Dentro del género Curvularia Boedijn, C. lunata (Wakker) Boedijn es la que se aísla con mayor frecuencia ${ }^{7}$.

Se presenta el caso clínico de una rinosinusitis alérgica por Curvularia inaequalis (Shear) Boedijn. Además se discuten los factores de riesgo, el diagnóstico y tratamiento de esta infección.

\section{Caso clínico}

Paciente varón de 56 años, residente de Olmué (Región de Valparaíso), con antecedente de rinitis alérgica crónica desde la juventud y rinosinusitis micótica diagnosticada y tratada con aseo quirúrgico endoscópico y fluconazol oral dos años antes. Consultó por cuadro de secreción nasal abundante y espesa, de color amarilla verdosa y de mal olor, con descarga posterior, cefalea y disminución del olfato. Se le realizó una tomografía axial computada (TAC) de senos paranasales que mostró un engrosamiento de la mucosa del seno frontal derecho y velamiento de los senos maxilar y etmoidal ipsilaterales (Figuras 1, 2 y 3). Se realizó una cirugía endoscópica que incluyó erradicación de las lesiones endonasales, etmoidectomía derecha amplia y meatotomía media con abordaje por vía Caldwell-Luc del receso maxilar derecho para asegurar la estricta limpieza y desfocación, obteniéndose abundante material (mucina) que fue enviado para estudio microbiológico.

Los cultivos bacterianos fueron todos negativos. Al examen microscópico directo con $\mathrm{KOH} 20 \%$ y con tinción de Gomori-Grocott (Figura 4) se visualizaron abundantes hifas septadas en ángulo dicotómico de $45^{\circ}$. En los cultivos en agar Sabouraud hubo desarrollo de Curvularia sp. El paciente fue tratado con itraconazol, en cápsulas de 400 $\mathrm{mg}$ al día por seis meses y corticoesteroides inhalatorios. Evolucionó con buena respuesta clínica. Actualmente se encuentra asintomático y realizando todas sus actividades habituales.

\section{Identificación de la especie}

Las muestras de mucina fueron sembradas en agar Sabouraud a 27 y $37^{\circ} \mathrm{C}$, obteniéndose en todos los cultivos
Universidad de Valparaíso, Chile.

Cátedra de Micología (RC). Hospital Naval de Viña del Mar, Unidad de Infectología (EB). Servicio de Otorrinolaringología (JE)

Los autores declaran no tener conflictos de interés.

Financiamiento: Trabajo financiado con recursos del Laboratorio de Micología Médica y Ambiental de la Universidad de Valparaíso.

Recibido: 20 de diciembre de 2012 Aceptado: 28 de marzo de 2012

Correspondencia a: Rodrigo Cruz Choappa rcruzchoappa@gmail.com 


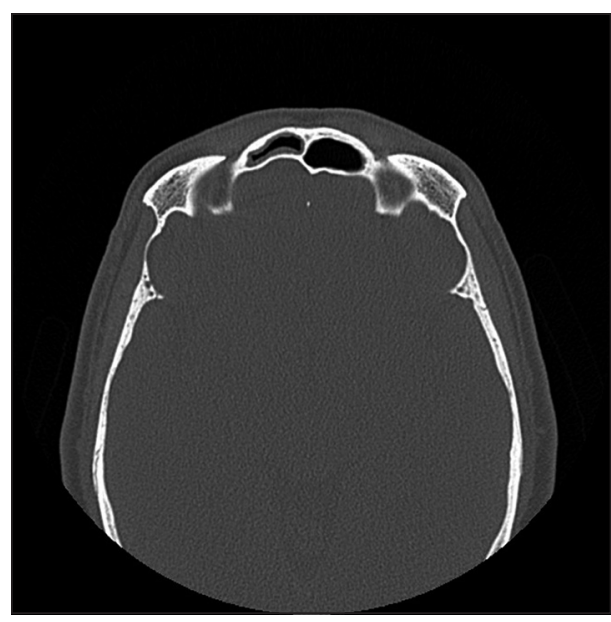

Figura 1. TAC de senos paranasales con engrosamiento mucoso del seno frontal derecho.

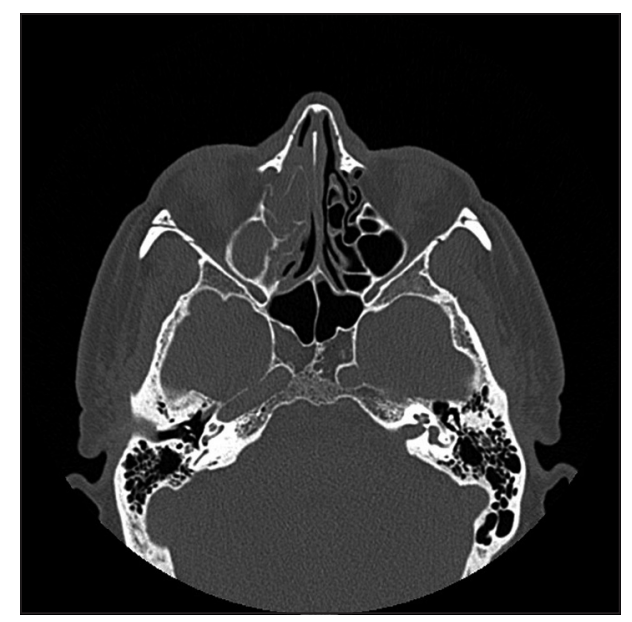

Figura 2. TAC de senos paranasales con velamiento difuso de las celdillas etmoidales derechas.

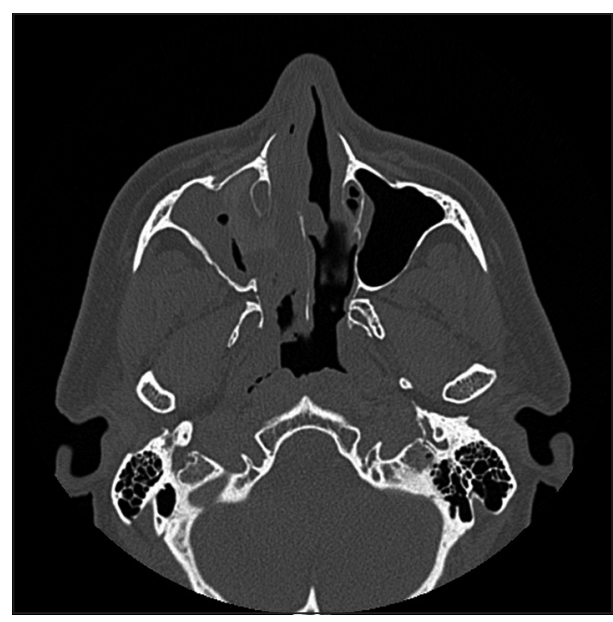

Figura 3. TAC de senos paranasales con velamiento del seno maxilar derecho. colonias filamentosas de color gris oscuro, con conidios secos. A los 5 días de incubación.

Luego fueron sembradas en agar harina de maíz y agar agua con paja de trigo a $25^{\circ} \mathrm{C}$ durante 10 días.

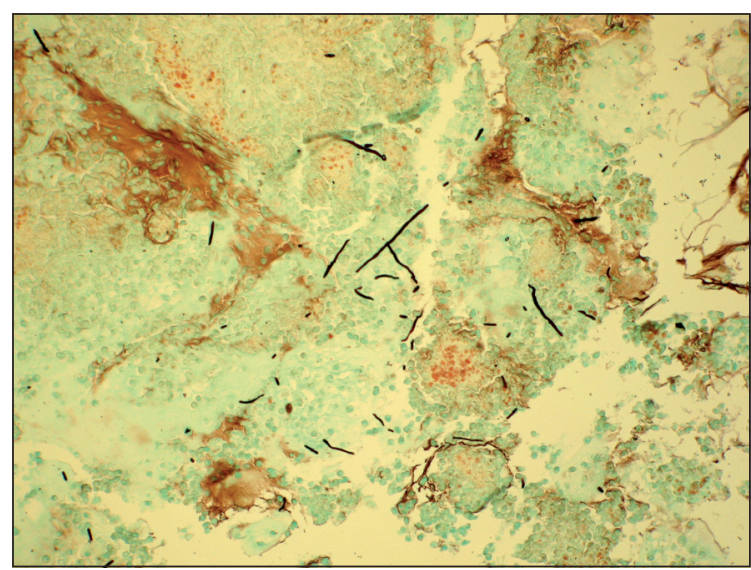

en ángulo dicotómico. Tinción Gomori-Grocott. 40X.

Figura 5. Macroconidios con célula central más larga, con 3-5 distoseptos. Tinción de lactofenol con azul de algodón. 100X.
Macroscopia: las colonias fueron de aspecto aterciopelado con centro algodonoso, de color gris oscuro en el anverso y negro en el reverso, con conidios secos abundantes.

Microscopia: se observaron hifas dematiáceas, conidióforos más o menos erectos, conidios con célula central más larga que las otras células, con 3-5 distoseptos y medidas de estos: 28-45 x 10-16 $\mu \mathrm{m}$ (Figura 5).

La descripción antes señalada coincide con la especie Curvularia inaequalis (Shear) Boedijn ${ }^{8,9}$.

\section{Discusión}

La RSFA corresponde a una entidad cuya patogenia y tratamiento ha motivado gran interés en el último tiempo. Su prevalencia se estima entre 4 y $10 \%$ de los pacientes con rinosinusitis crónica ${ }^{6,10,11}$. En esta patología los hongos provocan un cambio antigénico inicial en la superficie de la mucosa nasal y desencadenan reacciones de tipo I y tipo III $^{11,12}$. Estos fenómenos llevan a un edema tisular, inflamación crónica de la mucosa, infiltración de eosinófilos, linfocitos y formación de cristales de Charcot-Leyden. Todo esto favorece la formación de un material viscoso, denominado mucina alérgica, que rellena los senos paranasales comprometidos, impidiendo el drenaje natural e iniciando la autoperpetuación del proceso inflamatorio ${ }^{11-14}$.

Los signos clínicos considerados para la sospecha diagnóstica son el drenaje sinusal anterior o posterior, obstrucción nasal, presión, dolor facial y disminución del sentido del olfato ${ }^{3,6,11}$. En nuestro paciente se presentaron todos los signos nombrados, además de la inflamación de la mucosa nasal.

Schubert y Goetz ${ }^{4}$ propusieron cuatro criterios diagnósticos para la RSFA, los que incluyen: presencia de 
mucina alérgica como hallazgo quirúrgico o histopatológico, presencia de hifas en mucina alérgica en examen microscópico directo o desarrollo de hongos filamentosos en los cultivos, ausencia de invasión histopatológica del hongo y exclusión de otras formas de rinosinusitis fúngica. En nuestro caso, los cuatro criterios diagnósticos estuvieron presentes.

La TAC es el examen imagenológico de elección, donde se puede observar signos como engrosamiento mucoso, velamiento difuso y múltiple, a menudo unilateral, pero también puede ser bilateral ${ }^{15,16}$, hallazgos también presentes en nuestro paciente.

La endoscopia cumple un rol fundamental en el diagnóstico, tratamiento y seguimiento del cuadro y permite etapificar la RSFA mediante los hallazgos en la mucosa rinosinusal, según los criterios propuestos por Kupferberg ${ }^{17,18}$.

La confirmación micológica se realiza mediante la visualización de hifas en el examen microscópico directo con $\mathrm{KOH} \mathrm{20 \%} \mathrm{o} \mathrm{tinción} \mathrm{de} \mathrm{Gomori-Grocott,}$ previa fijación de la muestra, ademas del cultivo en agar Sabouraud a $37^{\circ} \mathrm{C}^{12,13,18}$. En nuestro caso hubo presencia de hifas septadas tanto en la visualización con $\mathrm{KOH}$ al $20 \%$, como en la tinción de Gomori-Grocott de la mucina, además del abundante desarrollo de C. inaequalis (Shear) Boedijn en los cultivos.

Elevaciones de la IgE específica para múltiples hongos podría ser de ayuda en el diagnóstico de RSFA en aquellos pacientes con cultivos y exámenes histológicos negativos, pero en el caso del aumento de la IgG específica para hongos su utilidad permanece aún incierta ${ }^{19-21}$.

Se han descrito varios géneros asociados a RSFA, siendo los principales Curvularia Boedijn, Bipolaris Shoemaker, Alternaria Nees ex Fries, Aspergillus Micheli ex Link, entre otros ${ }^{6,11,18,22}$. Las especies del género Curvularia Boedijn son principalmente saprófitas y fitopatógenas, aisladas en áreas tropicales o subtropicales y asociadas a distintos sustratos orgánicos. De las 35 especies conocidas, C. lunata (Wakker) Boedijn,
C. pallescens Boedijn y C. geniculata (Tracy \& Earlr) Boedijn son ubicuas y se han descrito en infecciones como queratitis, sinusitis, peritonitis, endocarditis e infecciones diseminadas $^{22,23}$. Curvularia inaequalis (Shear) Boedijn se ha aislado escasamente en infecciones sistémicas, cutáneas ${ }^{24}$ y sólo existe una comunicación de un caso de rinosinusitis alérgica presentado anteriormente ${ }^{25}$.

El tratamiento de estos pacientes incluye el aseo endoscópico, que permite la remoción de la masa fúngica y la restauración de la ventilación de las cavidades y los corticoesteroides, los cuales reducen el edema de la mucosa y la tasa de recurrencia ${ }^{26-28}$. El uso de antifúngicos por vía sistémica es controvertido, aunque existen publicaciones que sugieren que su asociación podría contribuir a disminuir las recurrencias ${ }^{7,29}$. Algunos autores han sugerido agregar antifúngicos tópicos, pero no ha sido demostrada su efectividad ${ }^{30}$.

Es importante aumentar la sospecha de la RSAF, además de realizar un diagnóstico apropiado y un tratamiento oportuno para disminuir los riesgos de recidiva en estos pacientes.

\section{Resumen}

Curvularia inaequalis (Shear) Boedijn es un hongo dematiáceo, saprófito y fitopatógeno, presente principalmente en áreas tropicales y subtropicales, asociado a distintos sustratos orgánicos. Se ha identificado escasamente en infecciones sistémicas, cutáneas y sólo existe una comunicación de un caso de rinosinusitis alérgica descrito anteriormente. Presentamos el caso clínico de un paciente con una rinosinusitis alérgica fúngica por Curvularia inaequalis (Shear) Boedijn en cuyo diagnóstico se consideró los síntomas y signos clínicos, la TAC de senos paranasales y el cultivo de la mucina. El paciente fue tratado con un aseo quirúrgico por vía endoscópica, además del uso de corticoesteroides inhalatorios e itraconazol sistémico. Presentó una buena respuesta clínica, encontrándose asintomático a un año del tratamiento.

\section{Referencias bibliográficas}

1.- Millar J W, Johnston A, Lamb D. Allergic bronchopulmonary aspergillosis of the maxillary sinuses. Thorax 1981; 36: 710

2.- Lamb D, Millar J W, Johnston A. Allergic aspergillosis of the paranasal sinuses. J Pathol 1982; 137: 56.

3.- Schubert M S. Allergic fungal sinusitis. Otolaryngol Clin North Am 2004; 37: 301-26.

4.- Schubert M S, Goetz D W. Evaluation and treatment of allergic fungal sinusitis. I. Demographics and diagnosis. J Allergy Clin Immunol 1998; 102: 387-94.

5.- Manning S C, Holman M. Further evidence for allergic pathophysiology in allergic fungal sinusitis. Laryngoscope 1998; 108: 1485-96.

6.- Alvarez V C, Guelfand L, Pidone J C, Soloaga R, Ontivero P, Margari A, et al. Rinosinusitis alérgica fúngica causada por Curvularia sp. Rev Iberoam Micol 2011; 28 : 104-6.

7.- Schubert M S. Allergic fungal sinusitis: pathophysiology, diagnosis and management Med Mycol 2009; 47 (Suppl 1): S324-30.

8.- Piontelli E. El género Curvularia Boedijn. Piontelli E, editor. Manual de microhongos filamentosos comunes I, $1^{\mathrm{a}}$ ed. Valparaíso: Edición particular 2011, p. 225-30.
9.- Shear C L. New species of fungi. Bull Torrey Bot Club 1907; 34: 305-17.

10.- Ponikau J U, Sherris D A, Kern E B, Homburger H A, Frigas E, Gaffey T A, et al. The diagnosis and incidence of allergic fungal sinusitis. Mayo Clin Proc 1999; 74: 877-84.

11.- Fonseca X, Fernández F. Rinosinusitis fúngica alérgica: revisión. Rev Otorrinolaringol Cir Cabeza Cuello 2005; 65: 45-54.

12.- de Shazo R D, Swain R E. Diagnostic criteria for allergic fungal sinusitis. J Allergy Clin Immunol 1995; 96: 24-35.

13.- Kuhn F A, Swain R Jr. Allergic fungal sinusitis: diagnosis and treatment. Curr Opin Otolaryngol Head Neck Surg 2003; 11: 1-5. 
14.- Torres C, Ro J Y, el-Naggar A K, Sim S J, Weber R S, Ayala A G. Allergic fungal sinusitis: a clinicopathological study of 16 cases. Hum Pathol 1996; 27: 793-9.

15.- Manning S C, Merkel M, Kriesel K, Vuitch F, Marple B. Computed tomography and magnetic resonance diagnosis of allergic fungal sinusitis. Laryngoscope 1997; 107: 170-6.

16.- Mukherji S K, Figueroa R E, Ginsberg L E, Zeifer B A, Marple B F, Alley J G, et al. Allergic fungal sinusitis: CT findings. Radiology 1998; 207: 417-22.

17.- Kuhn F A. Role of endoscopy in the management of chronic rhinosinusitis. Ann Otol Rhinol Laryngol Suppl 2004; 193: 15-8.

18.- Pérez F, Opazo H, Cruz R, Piontelli E. Reporte clínico: diagnóstico endoscópico e histológico de aspergilosis sinusal no invasiva en paciente inmunocompetente. Bol Micol 2006; 21: 85-9.

19.- Stewart A E, Hunsaker D H. Fungus-specific $\mathrm{IgG}$ and $\mathrm{IgE}$ in allergic fungal rhinosinusitis. Otolaryngol Head Neck Surg 2002; 127:
324-32.

20.- Gandur A F, Casado H S. Sinusitis alérgica micótica. Arch Alergia Inmunol Clin 2008; 39: 15-21.

21.- Mabry R L, Manning S. Radioallergosorbent microscreen and total immunoglobulin $\mathrm{E}$ in allergic fungal sinusitis. Otolaryngol Head Neck Surg 1995; 113: 721-3

22.- Carter E, Boudreaux C. Fatal cerebral phaeohyphomycosis due to Curvularia lunata in an immunocompetent patient. J Clin Microbiol 2004; 42: 5419-23.

23.- Pimentel J D, Mahadevan K, Woodgyer A, Sigler L, Gibas C, Harris OC, et al. Peritonitis due to Curvularia inaequalis in an elderly patient undergoing peritoneal dialysis and a review of six cases of peritonitis associated with other Curvularia spp. J Clin Microbiol 2005; 43: 4288-92.

24.- Tanabe K, Seino M, Senda S. Superficial mycosis of the breast caused by Curvularia inaequalis. Eur J Dermatol 2010; 20: 658-9.
25.- Posteraro B, Scarano E, La Sorda M, Torelli R, De Corso A, Mulé A, et al. Eosinophilic fungal rhinosinusitis due to the unusual pathogen Curvularia inaequalis. Mycoses 2010; 53: 84-8.

26.- Quraishi H A, Ramadan H H. Endoscopic treatment of allergic fungal sinusitis. Otolaryngol Head Neck Surg 1997; 117: 29-34.

27.- Taj-Aldeen S J, Hilal A A, Schell W A. Allergic fungal rhinosinusitis: a report of 8 cases. Am J Otolaryngol 2004; 25: 213-8.

28.- Schubert M S. Allergic fungal sinusitis: pathogenesis and management strategies. Drugs 2004; 64: 363-74

29.- Rains B M, Mineck C W. Treatment of allergic fungal rhinosinusitis with high dose itraconazol. Am J Rhinol 2003; 17: 1-8.

30.- Ricchetti A, Landis B N, Maffioli A, Giger R, Zeng C, Lacroix J S. Effect of anti-fungal nasal lavage with amphotericin B on nasal polyposis. J Laryngol Otol 2002; 116: 261-3. 